\title{
Enhancements of Subsystems and Payloads for Small Satellites
}

\author{
J. Martín Canales Romero ${ }^{1}$ \\ Technical Consultant National University of Engineering, Lima, Peru
}

\begin{abstract}
In the next future Small satellites can be used as experimental platforms improving their capabilities. It has been demonstrated that nanosatellites or picosatellites using the technology of CubeSats are being developed by different universities around the world. Which missions are possible to execute or which payloads can be installed in a such a satellite platform? Small satellites are conceived as dedicated systems to execute experiments or tests in space. We can compare a small satellite platform with others systems. A Drop Tower will be used to create some seconds (aprox. 10s) of microgravity. With Sounding Rockets the generated microgravity will take some minutes $(\max 14 \mathrm{~min})$. With a small satellite platform the microgravity condition will result in months or longer! From this point of view small satellites have obviouslly much advantage than a drop tower or a sounding rocket by executing experiments in microgravity. For sure the others systems have also advantages like an intact experimental pay load (Drop Tower), or the recovery of the payload and recorded data in short time just after the flight (Sounding Rocket) but for a small satellite this system functionality can also be developed, so that the payload can be also recovered intact or the data provided to ground as quick as possible. Peruvian engineers are working on a next generation of subsystems and payloads to be implemented in such experimental platforms. They are modelling and improving the capabilities of some, used in sounding rocket systems, importants instruments which can be carried in the proposed small satellite Platform. The instruments must be simplified, reduced in size, adapted and designed to properly perform their functions in the designed platform. All the subsystems will continually be designed for making them more efficient in order to use best possible the limited resources available in the satellite. Depending of the mission a next generation of pico satellites can emerge.
\end{abstract}

\section{Introduction}

$\mathrm{M}$ ICROGRAVITY is the environment where the acceleration generated by gravity is reduced to a minimum. The gravity field does not change but with help of another force it is possible to compensate this acceleration and "eliminate" it, producing microgravity.

Physics in microgravity environment is a very new field of science. This knowledge is possible to adquire only due experiments in weightlessness. On the earth it is not so easy to create a microgravity environment for extensive research. Only space flights and missions do provide the conditions for an appropriate microgravity laboratory.

There are three different methods to generate a microgravity environment:

1) One is to travel far away from the Earth to obtain a microgravity environment with low disturbances.

2) Another method is the free fall. Here the acceleration generated by gravity will be compensated with the acceleration of the body by falling.

3) The third method is orbiting the Earth. This is a kind of free fall and in this case the free fall is constant. The acceleration generated by gravity is cancelled due to the tangential acceleration.

A very good example of a microgravity laboratory is the International Space Station (ISS). Onboard the ISS many experiments using the conditions of microgravity are going on. Research institutes and companies are making experiments in physics, biology, fluids science, etc. The ISS is a very expensive project and the achievement of this kind of experiments is very costly. The ISS is a unique laboratory for long time investigations. In some cases experiments do not need to hold a microgravity environment for a long time but the ISS gives until now the unique

${ }^{1}$ Columbus Operations Coordinator, ESA Flight Control Team, German Space Operations Center (GSOC), Martin.Canales@dlr.de, AIAA Member Grade for first author. 
opportunity for performing science. In such cases it is possible to use other methods to generate microgravity, like a drop tower and sounding rockets. But all these experiments could be executed in optimal case in a laboratory with constant weithlessness like in space stations or satellites.

The intention of this paper is to promote the use of nanosatellites and picosatellites for microgravity research giving the opportunity to several educational institutions to play an important role in this scientific field. Also developing this kind of system will facilitate and accelerate the design of a very efficient and powerful system for microgravity research and contribute for a better future of the humanity.

\section{Space Laboratory ISS (constant microgravity environment)}

The gravity at the ISS is approximately $90 \%$ of the gravity at see level. For this reason the ISS is the best laboratory for microgravity research at the moment. Physical science plays a very important role for the planned experiments on the ISS. It is possible to develop new methods for manufacturing new kind of metals and semiconductors. By melting in microgravity is possible to eliminate interfering forces producing an homogeneous product. It is also possible to measure the surface tension and viscosity of liquids. The growth of crystals in the weightlessness affords the rearing of crystals without impurities. The biological experiments represent another important field in the microgravity research at the ISS. Bilogical experiments with plants and small organism are ongoing.

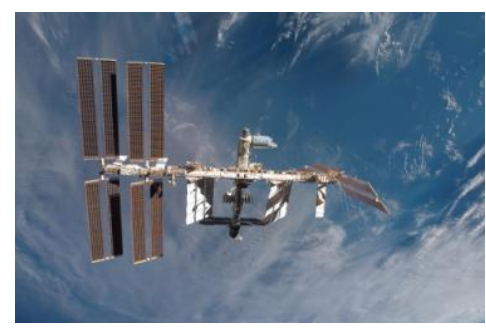

Figure 1. ISS space laboratory.

The European contribution to the ISS, the Columbus laboratory, has the following objetives for microgravity research:

1) Gravitational biology: to study the influence and the perception of gravity by organisms (humans, animals and plants)

2) Radiation and astrobiology: to find the impact of cosmic radiation in the human body

3) Material research: to optimize existing metallurgic processes to manufacture metals and semiconductors materials

4) Fluid physics: to have a better understanding in transport of liquids and gases for their use in space applications.

5) Combustion research: to analyze the combustion processes of liquid fuels under high pressure

6) Fundamental physics: to generate more efficient methods to produce electronic components (solar cells, microchips) using plasma.

\section{Drop Tower (microgravity lower than $10 \mathrm{sec}$ )}

In 1782 the English plumber William Watts observed by dropping molten plumb on the air far enough from the ground that perfect round balls were formed. He patented the process and used it to manufacture lead shots. This process is known as the Tower Casting Method. The manufacturing of lead shots with this process was achieved in a tower $40 \mathrm{~m}$ of altitude. The method consists to melt plumb $\left(400^{\circ} \mathrm{C}\right)$ at the top of the tower and cast it through round holes in a metal plate (colander). A water bath was placed on the ground where the solied balls were cooled. By the free fall the molten lead becomes spherical due to the influence of the surface tension. This manufacturing process guarantees perfect round lead shots. The Tower Casting Method uses a microgravity environment generated by free fall. The drop tower method generates microgravity using the same principle of the Tower Casting Method. The drop tower consists in a tube inside of the turm where the air is evacuated. With the creation of vacuum is possible to eliminate the aerodynamic resistance. Inside the tower a drop capsule contents the experiment which will experience a microgravity environment for a few seconds. Fig. 2 shows the drop tower located in Bremen, Germany, which belongs to the Center of Applied Space Technology and Microgravity at the University of Bremen. The tower is $123 \mathrm{~m}$ high and in free fall weightlessness for about 5 seconds

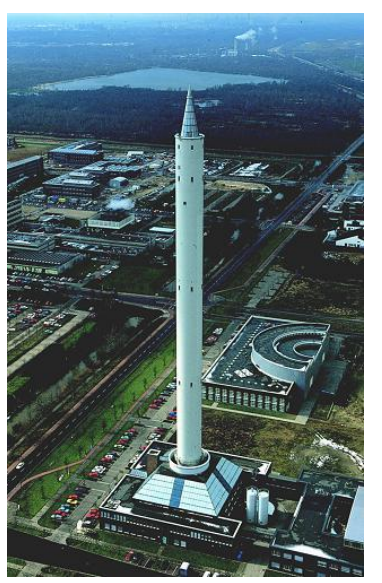

Figure 2. Drop Tower. can be generated. Using a catapult that accelerates the drop capsule (up to $500 \mathrm{~kg}$ ) to a speed of $48 \mathrm{~m} / \mathrm{s}$ the weightlessness time will be extended up to $9.5 \mathrm{sec}$. There it is possible to execute experiments up to three times a day.

The Japanese Laboratory for Microgravity uses a vacuum tube installed on the ground (100m depth). A high quality microgravity environment is created for about 4.5 seconds. The payload can weigh a maximum of $370 \mathrm{~kg}$. 


\section{Sounding rockets (microgravity from $4 \mathrm{~min}$ up to $14 \mathrm{~min}$ )}

Sounding rockets are a very atractive platform for microgravity experiments because it easily allows planning an experiment, to fly it and to get the experiment data results in a short time. With the appropiate infraestructure several launches per year can be achieved. Sounding rockets are an economical method to generate microgravity comparing with space stations or satellite platforms. Because of the series of sounding rockets campaigns the development of a large number of experiments and equipment was possible. This make a sounding rocket platform very flexible where late experiment changes can be executed at any time and also shortly before the launch. This is a very important advantage for biological experiments, which use live cells and organisms. A sounding rocket flight can generate microgravity between 4 min and up to maximal 14 minutes. This span of time is much better than the achieved by a drop tower but also not enough for extensive microgravity research.

Below are listed examples of experiments performed in sounding rockets missions:

1) Life Science

- Influence of gravity in single cells

- Examination of intercellular transfer of signals

- Metabolism of plants cells

2) Physical Science

- Solidification process of metal and alloys

- Growth of single crystals of semiconductors

- Growth of protein crystals

- Fluid-dynamic and diffusion experiments

- Investigation of boiling and evaporation

- Colloidal chemistry experiments

- Combustion experiments

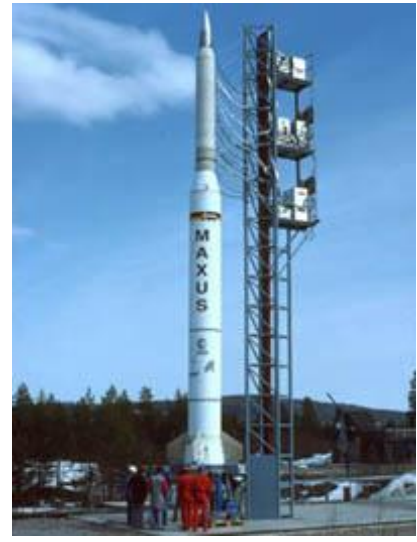

Figure 3. Sounding rocket

\section{Others}

It is important to mention other methods that are being used to create microgravity. Those are:

\section{A. Parabolic flight (microgravity up to 20 sec)}

Parabolic flights are used to conduct microgravity for short time periods (up to $20 \mathrm{sec}$.). In a flight campaign the aircraft will fly several times parabolas. This method is used for the training of astronauts, for conducting investigations in physical and life science, for testing instruments to be flown in space missions, and also to film space scenes, etc. The advantages of parabolic flights are:

1) Short lead Time

2) Low cost

3) Flexible experimental approach

4) Direct intervention of the scientists

5) Modification of the experiment between flights

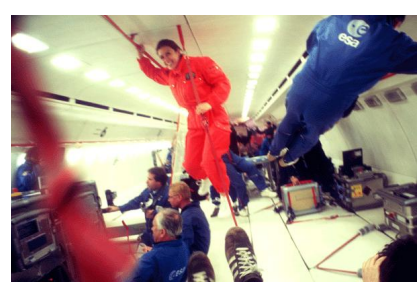

Figure 4. Parabolic flight

\section{B. Balloon-based Drop Capsule (30 sec of useful microgravity)}

The Japanese Space Agency (JAXA) has also a program for microgravity research. In this program a microgravity environment is created with the support of a balloon, which carries an experiment-capsule. The capsule will be dropped from the balloon at the altitude of $40 \mathrm{~km}$. The capsule falls 1 minute long and of this time, 30 seconds of microgravity environment for the experiments on board will be provided. The payload can weigh up to $300 \mathrm{~kg}$. The drop capsule is built as an aerodynamic vehicle and has the shape of a small rocket. The capsule consists of two parts, the shell which protects the payload from aerodinamical turbulences and the payload which is floating inside the shell (no mechanical contact exists between both). The microgravity environment is reached due to the free fall effect.

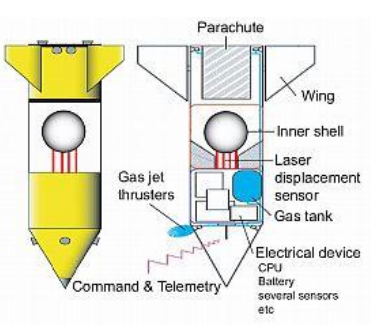

Figure 5. Drop capsule 


\section{Small satellites}

Other method to generate microgravity is orbiting the Earth. The Russian Foton satellites performed with success several microgravity experiments in biology and material science. The satellite is a derivation of the Vostok capsule and weighted over six tons. One advantage of the Foton missions was the avoidance of rotating parts which can disturb and reduce the quality of the created microgravity environment. Due to the usage of a re-entry system the recovery of the payloads was another benefit.

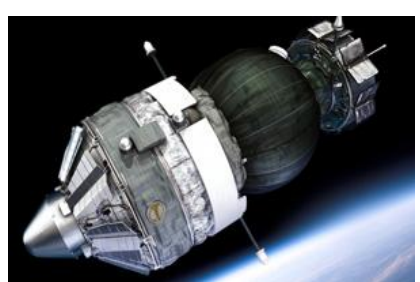

Figure 6. Foton vehicle

The advantage of satellites platforms in the microgravity research is that a permanent microgravity environment is always available. Such a platform can achieve more accurate experiment results and assure the generation of more science data. To intensify the microgravity research is necessary to exploit the growing development of nano and pico satellite. Nowadays the development of these satellites is economical and also the launch cost is very low (piggy-back). This kind of missions can also be cheaper than sounding rockets campaigns. To guarantee the success of a mission the development of a good and efficient platform is indispensable and unavoidable. Universities can help in the design of these platforms and their instruments. A joint venture between the industry and universities allow the conception and test of new space products. Research institutions can provide and support with more flexible and economical tools.

Taking into account the above mentioned points small satellites platforms are very useful:

1) The time-life of the satellite depends on the mission itself. The orbit will define how long the satellite will orbit the Earth. It is also possible to plan very short missions.

2) Instruments designed for sounding rockets can be adapted and installed in satellites to avoid extra investments.

3) A small spacecraft in a high orbit can offer a very good quality microgravity environment. A high orbit avoids disturbances available in low orbit platforms like the ISS.

4) New methods and strategies can be tested in a short time:

- Mechanisms can help to deploy solar cells, antennas, etc

- Small tantalum shields can protect sensible components against the radiation

- Strengthen structures of titanium can protect the spacecraft electronic, etc

5) The modern electronic components allow the design of low power electronics. The components are subsequently cheaper and tolerate a broad temperature range. Furthermore, a reduction of the platform size and the weight of the modules. Components that are not certified for space applications can be used, what is surely not allowed in commercial satellites.

Currently Peruvian universities are working on the design of low power small satellite platforms. The objective driving this work is the development of a high efficient system to offer the best performance for microgravity experiments. This platform or bus will tested in the framework of the QB50 project. Furthermore the implementation of a ground stations network (UHF antennas) to support this project is being designed. Another project to set up a S-Band network is in plan.

\section{Equipment and Components for Small Spacecrafts}

This section is dedicated to examples of equipment and components that can be used in small spacecrafts

\section{A. Silver Oxide Batteries}

For space applications several types of batteries have been used:

1) Silver-Zinc battery is one of the first used for space applications (in the 60's). This battery has a very high specific power and energy. The disvantage of this battery is its high price.

2) Nickel-Cadmium batteries were the most popular batteries used on the 70's for space applications. This battery still in use and is the most common for LEO satellites.

3) Nickel-Hydrogene battery is currently the most popular battery for space applications. The life-time of these batteries can reach up to 20 years. A disadvantage is that this battery is also very expensive.

4) Lithium Ion batteries are very light and are used often for space missions.

5) Sodium-Sulfur batteries were already used in space. This battery has a high energy density, long life and low price. The disvantage is that the battery can explote in contact with water.

6) Silver-oxide batteries have long life and very high energy/weight ratio. Due to the use of silver the cost is very high. It provides up to 40 percent more run time than the lithium-ion batteries. In the Apollo program these batteries were introduced the first time for space applications. These batteries can be used in

4

American Institute of Aeronautics and Astronautics 
experiments which need high energy consumption for a short time or in systems without solar cells. This makes the silver-oxide batteries very attractive for small satellites.

\begin{tabular}{|l|c|c|c|}
\hline & Silver-oxide battery & Lithium-ion battery & $\begin{array}{c}\text { Nickel hydrogen } \\
\text { battery }\end{array}$ \\
\hline Energy density, Wh/kg & 130 & $100-160$ & 75 \\
\hline Energy/size, Wh/L & 500 & $250-360$ & 55 \\
\hline Power/weight, W/kg & High & $250-340$ & 220 \\
\hline Charge/discharge efficiency, \% & N/A & $80-90$ & 85 \\
\hline Time durability & High & $(24-36)$ months & N/A \\
\hline Cycle durability, cycles & N/A & $\sim 1200$ & 20,000 \\
\hline
\end{tabular}

Table 1. Batteries parameters.

\section{B. Transmitter Modules}

To dump large quantities of data a mini S-Band transmitters can be used or also in some cases, by a small energy budget, one or more UHF transmitters. This method is used in sounding rockets which can transmit data in parallel in different frequencies, so that the transmission bandwidth is multiple.

\begin{tabular}{|l|c|c|c|c|c|}
\hline & S Band & $\mathbf{1 x}$ & $\mathbf{2 x}$ & $\mathbf{3 x}$ & $\mathbf{4 x}$ \\
$\mathbf{U H F}$ & $\mathbf{U H F}$ & $\mathbf{U H F}$ & $\mathbf{U H F}$ \\
\hline Data Rate, Kbit/s & Up to 115 & 9,4 & 18,8 & 28,2 & 37,6 \\
\hline Power Consumption, mW & 2000 & 300 & 600 & 900 & 1200 \\
\hline Weight, gr & 125 & 70 & 140 & 210 & 280 \\
\hline
\end{tabular}

Table 2. Transmitters for CubeSats.

\section{Microcontroller}

The new generation of low power microcontrollers and electronic components permit the design of low power modules, making the platform more efficient and reducing the power budget of the satellite.

\begin{tabular}{|l|c|c|}
\hline & $\begin{array}{c}\text { MSP430F5XX } \\
\text { Texas } \\
\text { Instruments }\end{array}$ & MC9S08XX \\
A. Freescale \\
\hline Supply-Voltage, V & $1,8-3,6$ & $1,8-3,6$ \\
\hline Power consumption active, micro Ampere & 200 & 185 \\
\hline Power consumption stand-by, micro Ampere & 2,5 & 7,3 \\
\hline System clock, MHz & Up to 25 & 20 \\
\hline CPU Bus, bit & 16 & 8 \\
\hline
\end{tabular}

Table 3. Low power microcontrollers.

\section{LED and Lasers Diodes}

A new generation of high brightness and low power LEDs can be introduced as light sources for experiments. A LED consumes normally $30-60 \mathrm{~mW}$. The available colors are infrared, red, orange, yellow, green, blue, violet, purple, ultraviolet and white.

The advantages of these LED are its efficiency, i.e. produces more light per watt; its color, emiting light in different wavelengths; its size (smaller than $2 \mathrm{~mm}$ ); and its shock resistance.

The laser diodes have wavelengths of $375-1625 \mathrm{~nm}$. The low power laser diodes consume $3-5 \mathrm{~mW}$ and have wavelengths of $635-670 \mathrm{~nm}$. On the market laser diodes in red, green, blue and violet colors area available. 


\section{Conclusion}

The microgravity research can intensively be supported from educational institutions and academia. Through their involvement complex systems and new instruments can easily be designed and developed. Moreover the development costs decrease. This cooperation will benefit universities, industry and research institutions. The challenge is to integrate academia and industry in this research field.

\section{References}

${ }^{1}$ Willi Hallman, Wilfried Ley, Klaus Wittmann ,Handbuch der Raumfahrttechnik, 3 rd ed., Hanser Fachbuch, Munich, 2007, Chap. 7.

${ }^{2}$ E. Messerschmid, S. Fasoulas, Raumfahrtsysteme, 3rd ed., Springer Verlag, Munich, 2008.

${ }^{3}$ H. Roeser, R. Sandau, A. Valenzuela, Small Satellites for Earth Observation, 1st ed., Gruyter, Berlin, 2005.

${ }^{4}$ R. Monti, Physics of Fluids in Microgravity, 1 st ed., Routledge, Florence, 2001.

"ZARM Drop Tower Bremen" [online database], URL: http://www.docstoc.com/docs/24240424/ZARM-Drop-TowerBremen-User-Manual.pdf

"REXUS User Manual," [online database], URL: http://rexusbexus.net/images/stories/rexus/rx-rexus user manual-v7 004dec09.pdf 